\title{
Spatial biases in understanding descriptions of static scenes: the role of reading and writing direction
}

\author{
Antonio Román • Abderrahman El Fathi • Julio Santiago
}

Published online: 10 January 2013

(C) Psychonomic Society, Inc. 2013

\begin{abstract}
Prior studies on reasoning tasks have shown lateral spatial biases on mental model construction, which converge with known spatial biases in the mental representation of number, time, and events. The latter have been shown to be related to habitual reading and writing direction. The present study bridges and extends both research strands by looking at the processes of mental model construction in language comprehension and examining how they are influenced by reading and writing direction. Sentences like "the table is between the lamp and the TV" were auditorily presented to groups of mono- and bidirectional readers in languages with left-to-right or right-to-left scripts, and participants were asked to draw the described scene. There was a clear preference for deploying the lateral objects in the direction marked by the script of the input language and some hints of a much smaller effect of the degree of practice with the script. These lateral biases occurred in the context of universal strategies for working memory management.
\end{abstract}

Keywords Reading and writing direction - Spatial bias ·

Mental model $\cdot$ Flexibility $\cdot$ Working memory ·

Bilingualism · Language comprehension

How is linguistic content represented such that people can reason about it? And how is it affected by habitual reading and writing direction (RWD)? One theory of language

\footnotetext{
A. Román $(\bowtie) \cdot J$. Santiago

Department of Experimental Psychology, University of Granada, Campus de Cartuja s/n,

18071 Granada, Spain

e-mail: reche@ugr.es

A. El Fathi

Department of Hispanic Philology, Université Abdelmalek Esaadi, Morocco, Tetouan, Morocco
}

comprehension that has received strong support in the literature is the theory of mental models (Johnson-Laird, 1983; see Van Dijk, \& Kintsch, 1983, for a related proposal; see Zwaan \& Radvansky, 1998, for a review). Mental models are working memory representations about situations and events in the world. They are analogical, spatial, and populated by concrete content, although they can also represent abstract or temporal content (Goodwin \& Johnson-Laird, 2005; Santiago, Román, \& Ouellet, 2011).

The most important feature of mental models is that they allow us to represent and manipulate reality in working memory in order to make decisions and deal with the situation. Working memory is characterized by limited capacity and effortful processing. Therefore, people tend to create only one such mental model, integrating into it all the information that is relevant to solving the problem at hand. The greater the amount of information that must be kept simultaneously in working memory, the more difficult the resolution of the problem (Goodwin \& Johnson-Laird, 2005).

Under this view, language serves to provide instructions that guide mental model construction in the comprehender (Johnson-Laird, 1983). From a linguistic input such as "the table is between the lamp and the TV," the listener can construct a mental model that represents the spatial position of those three objects. Although many different spatial arrangements are consistent with the statement, people tend to create a single model that captures only one spatial configuration. Many studies show that, in a task like this, the preferred mental model places the mentioned objects in a linear array, either a horizontal or a vertical one (see Evans, Newstead, \& Byrne, 1993, for a review).

Jahn, Knauff, and Johnson-Laird (2007) studied how mental models are used to reason about such static spatial configurations. They presented sets of descriptions of scenes and asked participants to judge whether the set was consistent or not. To use their same example, 
A table is between the TV and a chair.

The light is on the left of the TV.

The table is next to the light.

They predicted that people tend to make a single mental model of the first statement and then try to integrate the next two into it. Depending on the configuration of the initial model, the integration may be easy or difficult, as in the example above. In this example, the descriptions are consistent if, in the initial model, the chair is placed to the left of the table. Otherwise, participants will tend to claim that the premises are inconsistent or will take longer to find the correct answer.

Jahn et al. (2007) postulated that the preferred initial model should include the three mentioned objects in leftto-right $(\mathrm{L}-\mathrm{R})$ order. For the example above, a schematic model of this kind would be:

TV table chair.

This assumption was strongly supported by the data. Jahn et al. suggested that this preference for $\mathrm{L}-\mathrm{R}$ models was a bias induced by the habitual RWD. One central goal of the present article is to directly assess the role of RWD in mental model construction from linguistic descriptions such as those used by Jahn et al.

Available evidence suggests that habitual RWD exerts subtle but consistent effects on a wide variety of mental processes and representations, taking the form of lateral biases that follow the direction of the script (see Santiago \& Román, 2012, manuscript submitted for publication). For example, it has been shown that RWD is able to affect lowlevel skills such as word reading (Mishkin \& Forgays, 1952), perceptual span (Pollatsek, Bolozky, Well, \& Rayner, 1981), lateral motion perception (Maass, Pagani, \& Berta, 2007), attention (Spalek \& Hammad, 2005), exploration (Chokron \& Imbert, 1993; Kugelmass \& Lieblich, 1970), and hand movements in copying and drawing tasks (Nachshon, 1985; Shanon, 1979). More interesting for current concerns, RWD effects have been observed on the mental representation of highly abstract concepts, such as number magnitude (Dehaene, Bossini, \& Giraux, 1993; Zebian, 2005), time (Fuhrman \& Boroditsky, 2010; Ouellet, Santiago, Israeli, \& Gabay, 2010; Tversky, Kugelmass, \& Winter, 1991), events (Dobel, Diesendruck, \& Bölte, 2007; Maass \& Russo, 2003), letter sequences (Shaki \& Gevers, 2011), and social groups differing in agentivity (Maass, Suitner, Favaretto, \& Cignacchi, 2009). RWD is also able to bias the choice of behavioral alternatives (such as study items) from a list (Ariel, Al-Harthy, Was, \& Dunlosky, 2011). Even the aesthetic choices of artists, as well as the feelings that are aroused by pieces of visual art, can be affected by RWD (Chokron \& De Agostini, 2000; Nachshon, Argaman, \& Luria, 1999; Pérez González, 2011).
The standard account of RWD effects suggests that practice in reading and writing a given script induces directional habits that grow slowly and become progressively entrenched (Nachshon, 1985). These habits induce lateral biases in those skills that are components of the tasks of reading and writing (such as exploring the page or programming precise hand and finger movements). Moreover, as the eyes or hands move along the text, the mind moves along the concepts that are referred to by the text, opening the door to specific directional biases in their mental representation. Take temporal order as an example. Following universal pragmatic principles (Grice, 1975), events are usually described in the same order as that in which they occurred. Thus, the reader is exposed to a quite consistent correlation between word order along the horizontal axis and the temporal order of events. This correlation is hypothesized to cause the tendency to represent temporal sequences along a spatial horizontal axis in the mind, flowing in the same direction as the script (Santiago et al., 2011). A similar reasoning applies to numerical and other types of sequences. Because all languages studied in this context so far use a word order in which the syntactic subject precedes the syntactic object, and because subjects and objects canonically incorporate the thematic roles of agent and patient, the user of a script also experiences a correlation between thematic roles and left-right space, which is hypothesized to cause the tendency to represent agents preceding patients along the horizontal axis in the direction of the script (Maass \& Russo, 2003; Maass et al., 2009).

Under the standard account, when people are exposed to conflicting directional experiences (e.g., bidirectional readers of French and Arabic), they will develop contradictory directional habits that will enter into competition. As a result, lateral biases should be weaker than in people with fully consistent directional habits. Many published studies support this view (see, e.g., Kugelmass \& Lieblich, 1970, for exploration tasks; Nachshon, 1985, for reproduction tasks; Berch, Foley, Hill, \& Ryan, 1999, for numerical sequences; and Dobel et al., 2007, for agent-patient representation).

Thus, the standard account of high-level RWD biases suggests that they originate from experiences that occur during text comprehension, but there is very little evidence looking directly at RWD effects on mental model construction processes from linguistic input. Following up on work by Chatterjee, Southwood, and Basilico (1999), Maass and Russo (2003) asked their participants to draw sentences such as "the girl gives a gift to the boy" and found that Italians prefer to place the girl on the left and the boy on the right, whereas Arabs ${ }^{1}$ showed the opposite preferences. However, these results have not always been replicated:

\footnotetext{
${ }^{1}$ We herein use the term "Arabs" to mean "users of Arabic languages."
} 
Altmann, Saleem, Kendall, Heilman, and Rothi (2006) found that both English and Arabic readers tend to place the agent on the right, and Barrett, Kim, Crucian, and Heilman (2002) failed to observe any lateral bias in R-L versus L-R Korean readers. Moreover, all available evidence focuses on the representation of intrinsically sequential concepts (numbers, time, events), and it is unclear what will occur with static scenes. To our knowledge, the only relevant study is Geminiani, Bisiach, Berti, and Rusconi (1995). They presented descriptions of static and dynamic scenes to Italian students and found a strong tendency to place the first mentioned item on the left and the second item on the right. However, no R-L group was included, so the effects of RWD in such a task remain to be elucidated.

If the effects of RWD arise during mental model construction in language comprehension, they should also be present when descriptions of static scenes, fully devoid of any intrinsic directionality, order, or motion, are understood. Moreover, they should be reversed in readers of scripts with opposite directionality. The aim of the present investigation was to provide a first exploration of the influence of RWD on mental model construction avoiding any potential confounding with intrinsic order, thus bridging the gap between the literature on language comprehension and high-level effects of RWD. We set to do so in a way that would allow both an examination of the detailed processes of working memory management during language comprehension and a test for the effect of degree of conflicting habits due to practice with scripts of different directionality. We hypothesized that RWD would affect the directionality of mental model construction and that greater practice with a different RWD would lead to weaker, or even reversed, lateral biases. Independently of the effects of RWD, all groups would show a trend toward minimizing working memory load. In other words, all groups were expected to show a preference for minimizing the number of items and the time those items were kept in working memory before production. Thus, all groups were expected to show the same preference for producing items in the same order as that in which they were mentioned in the input sentence.

In order to do so, we devised a simpler version of Jahn et al.'s (2007) task. Participants were asked to listen to sentences such as "The table is between the lamp and the TV" and then draw the scene described by the sentence. Both order of drawing the mentioned objects and order of filling the spatial locations on the paper were measured. These measures allowed us to independently study spatial biases in the construction of mental models and working memory management processes (see details below). In order to assess the effect of RWD, in Experiment 1, we compared native Spanish users who read and write from left to right and native Arabic users from Morocco whose preferred script runs from right to left but are also familiar with $\mathrm{L}-\mathrm{R}$ scripts such as French or Spanish. In order to better evaluate the effect of conflicting RWD habits, we also included a group of native users of Arabic languages who had been living in Spain for a number of years and were highly acculturated into Spanish culture and language. Experiment 2 searched for the effect of the input language using a sample of bilingual bidirectional participants who performed the same task listening to the sentences in either an $\mathrm{L}-\mathrm{R}$ or an $\mathrm{R}-\mathrm{L}$ language.

\section{Experiment 1}

Method

\section{Participants}

There were three groups of participants. The Spanish group was composed of 21 psychology students at the University of Granada (mean age, 21 years; 5 males; 1 left-handed). All of them were native Spanish speakers, had never lived in an Arabic country for longer than an occasional stay, and did not know any R-L language.

The Moroccan group was made of 18 Moroccan students from the Abdelmalek Esaadi University, Tetouan, Morocco (mean age, 22 years; 12 males; none left-handed). Demographic information for 3 participants was lost due to a computer problem (which also affected information for 3 participants in the next group). All the remaining participants were born in Morocco and had never lived in a Western country. They all were native speakers of Moroccan Darija (the local Arabic dialect), and 9 of them were also native speakers of Standard Arabic (starting before the age of 4). Fourteen of them were also highly fluent in French, and 9 participants in this group also had some knowledge of Spanish (started in high school or university). All of them were highly proficient and habitual readers of Standard Arabic. ${ }^{2}$ The Moroccan group did the task in Darija.

The Arabs-in-Spain group was composed of 26 Arab students at the University of Granada (mean age, 22 years; 12 males; 1 left-handed). As was mentioned above, demographic information from 3 participants was lost. For the remaining sample, 18 were originally from Morocco, 3 from Jordan, 1 from Iraq, and 1 from Mauritania. Their average number of years living in Spain was 5.8 (range, 1-11). All of them were native speakers of at least one Arabic language (Standard Arabic, Moroccan Darija, Mauritanian Hassania, Jordanian Levantine, Iraqi Arabic). All of them were also native or highly fluent speakers of at least one European

\footnotetext{
${ }^{2}$ Standard Arabic is the only written Arabic language. Local Arabic languages are only oral (Lewis, 2009).
} 
language (mostly French and/or Spanish). All of them were highly proficient readers of Standard Arabic, and only 3 of them reported not reading it on a daily basis. All of them were highly fluent in Spanish and had no problems in understanding the instructions or having a conversation in Spanish with the experimenter. As is described in detail below, the Arabs-in-Spain group did the task in Spanish.

It is important to note that all participants in both Arab groups are bilingual (often multilingual), knowing at least one L-R language (modally French). The difference between them is not so much a difference of bilingualism, but of immersion in a particular language and writing system and extended experience with it.

The Spanish Group received course credit, and the two Arabic groups received a small gift or monetary compensation.

\section{Materials}

Five sentences were constructed, all of which consisted of an assertion that referred to a between relation among three different entities:

1. The table is between the lamp and the TV.

2. The bike is between the lamppost and the car.

3. The cup is between the bottle and the dish.

4. The pencil is between the book and the eraser.

5. The man is between the house and the tree.

We selected those entities because they are very common in both cultures, thereby avoiding comprehension problems or potential biases due to different degrees of familiarity. Importantly, all sentences refer to completely static scenes without any agentive structure. With the single exception of "the man" in the last sentence, all mentioned objects are inanimate entities, and they are embedded in sentences using a copulative verb. In the last sentence, "the man" is located in the center of the scene carrying out no action, and thus, it is unlikely that its animacy or agentivity may bias the location of the surrounding objects in the mental model in any particular direction.

\section{Procedure}

The participants were tested individually. They sat at a desk with a pile of five blank sheets and a pen. They were instructed that they would listen to five sentences describing scenes and that their task was to draw each scene on a different sheet. There was no mention of using a particular spatial arrangement (say, e.g., horizontal). The sentences were read aloud by the experimenter always in the same order, and coding was made in situ and out of the sight of participants. The Spanish and Arabs-in-Spain groups were tested at the University of Granada, Spain, and heard the instructions and sentences in Spanish. The Moroccan group was tested at the Abdelmalek Esaadi University, Morocco, and did the task in Darija. The order of mention of the objects in each sentence was the same in both languages.

\section{Data coding}

For each sentence, we measured the order in which each of the three mentioned objects was drawn (hereafter, this measure will be referred to as mentioned object order, or just object order) and the order in which the three positions (left, center, right) were filled (spatial order).

Each sentence presented auditorily the three objects in a temporal sequence or order of mention. In the sentence "the table is between the lamp and the TV," table was the first object mentioned, followed by lamp and then TV. Participants could draw the three objects in one out of six possible combinations of object order (see Table 1). For example, combination 213 meant that the first object to be drawn was the second object mentioned in the sentence (lamp), followed by the object mentioned first (table) and then by the object mentioned third (TV).

Regarding the order of location filling, or spatial order, there were also six possible combinations (see Table 1). For example, LCR meant that the object on the left was drawn first, then the central object, and then the object on the right. The combinations LCR, CLR, and LRC were grouped as patterns from left to right $(\mathrm{L}-\mathrm{R})$, and the combinations $\mathrm{RCL}$, CRL, and RLC were grouped as patterns from right to left (R-L).

The combination of each object order with one out of two possible spatial orders produced only one possible drawn model with the lateral objects (e.g., lamp and TV) in different positions (see Table 1 for details). To carry on with the example sentence "the table is between the lamp and the

Table 1 Mentioned object order and spatial order combinations and the resulting drawn models

\begin{tabular}{|c|c|c|c|c|}
\hline $\begin{array}{l}\text { Mentioned } \\
\text { object order }\end{array}$ & In our example & Spatial order & Pattern & $\begin{array}{l}\text { Drawn } \\
\text { model }\end{array}$ \\
\hline \multirow[t]{2}{*}{123} & \multirow[t]{2}{*}{ table-lamp-TV } & CLR & $\mathrm{L}-\mathrm{R}$ & lamp-table-TV \\
\hline & & CRL & $\mathrm{R}-\mathrm{L}$ & TV-table-lamp \\
\hline \multirow[t]{2}{*}{132} & \multirow[t]{2}{*}{ table-TV-lamp } & CLR & $\mathrm{R}-\mathrm{L}$ & TV-table-lamp \\
\hline & & CRL & $\mathrm{L}-\mathrm{R}$ & lamp-table-TV \\
\hline \multirow[t]{2}{*}{213} & \multirow[t]{2}{*}{ lamp-table-TV } & RCL & $\mathrm{R}-\mathrm{L}$ & TV-table-lamp \\
\hline & & LCR & $\mathrm{L}-\mathrm{R}$ & lamp-table-TV \\
\hline \multirow[t]{2}{*}{231} & \multirow[t]{2}{*}{ lamp-TV-table } & LRC & $\mathrm{L}-\mathrm{R}$ & lamp-table-TV \\
\hline & & RLC & $\mathrm{R}-\mathrm{L}$ & TV-table-lamp \\
\hline \multirow[t]{2}{*}{312} & \multirow[t]{2}{*}{ TV-table-lamp } & RCL & $\mathrm{L}-\mathrm{R}$ & lamp-table-TV \\
\hline & & LCR & $\mathrm{R}-\mathrm{L}$ & TV-table-lamp \\
\hline \multirow[t]{2}{*}{321} & \multirow[t]{2}{*}{ TV-lamp-table } & LRC & $\mathrm{R}-\mathrm{L}$ & TV-table-lamp \\
\hline & & RLC & $\mathrm{L}-\mathrm{R}$ & lamp-table-TV \\
\hline
\end{tabular}


TV," suppose that a participant showed a 213 (lamp-tableTV) object order and an RCL (right-center-left) spatial order. The resulting drawn model would have the lamp on the right side and the TV on the left (an R-L model). If the same object order was combined with an LCR spatial order, the resulting drawn model would have the lamp on the left side and the TV on the right side (an L-R model).

\section{Hypotheses}

We expected that habitual RWD would exert clear effects on the spatial order in which objects were drawn. Because we did not expect effects of RWD on object order (see below), RWD should also affect the final spatial configuration of the drawn model: Spanish participants would tend to draw L-R models, with the lamp on the left and the TV on the right, whereas Moroccan participants would prefer R-L models, with the lamp on the right and the TV on the left. A second hypothesis concerns the group of Spanish-acculturated Arabs: we expected that their extended practice with an L$\mathrm{R}$ language would make them show a weaker R-L bias or even switch to a $\mathrm{L}-\mathrm{R}$ bias, like Spanish participants.

Our third hypothesis follows from the fact that each object order imposes different costs on working memory resources. For example, the pattern 123 means that we draw the objects in the same order as that in which they appear in the speech stream. This pattern imposes the lowest memory load, because the objects are produced in the same order as that in which they are heard, thereby reducing the length of time they are held in memory. The pattern 231 imposes a greater memory load because it draws last the object presented first. Table 1 lists the combinations of object order from the one requiring fewer cognitive resources (123) to the one requiring the most (321). We expected that all the groups would prefer to use object orders that required fewer memory resources. For each one, the $\mathrm{L}-\mathrm{R}$ spatial order would be preferred by Spanish participants, and the R-L spatial order would be preferred by Moroccans, with the Arabs-in-Spain group somewhere in between.

It should be emphasized that, although tempting, the directionality of the drawn model cannot be taken as an index of the directionality of referents in the underlying mental model without a careful assessment of working memory management that ensures that all groups follow the same general principle of minimizing memory load. Once this is secured, drawn model directionality can be confidently used as an index of mental model directionality.

\section{Results}

If any central entity (e.g., the table) was drawn anywhere else than the center, the trial was considered invalid and was not included in the final analysis. We also excluded those items drawn vertically or somehow differently from the horizontal axis. In all groups, there were participants who saw all five of their trials rejected as invalid trials, which amounted to the rejection of the participant altogether. This occurred to 2 participants in the Spanish group, 1 in the Moroccan group, and 1 participant in the Arabs-in-Spain group. Therefore, effective sample sizes were 19, 17, and 25 , respectively. In the remaining participants, the average number of items rejected was $6.1 \%(7.2 \%, 5.3 \%$, and $5.9 \%$ in the Spanish, Moroccan, and Arabs-in-Spain groups, respectively).

In the analysis of drawn models, there were significant differences among the groups in the proportion of valid $\mathrm{L}-\mathrm{R}$ trials, $F(2,58)=5.52, p=.006, \eta^{2}=.16$ (see Table 2 for cell means and standard errors). The Spanish and Moroccan groups were significantly different from each other (70.7 \% vs. $38.3 \%$, respectively), $t(1,34)=3.70, p<$ .001. Consistent with the findings of Jahn et al. (2007) with German participants, Spanish participants preferred to represent the mentioned objects from left to right (with the lamp on the left and the TV on the right). In contrast, Moroccan participants showed the opposite bias (see Fig. 1). The Arabs-in-Spain group did not differ from the Spanish group $(58.8 \%$ vs. $70.7 \%), t(1,42)=-1.25, p=$ .21 , whereas they differed from the Moroccan group $(58.8 \%$ vs. $38.3 \%), t(1,40)=2.17, p=.03$. Thus, the Spanish-acculturated Arabs behaved more like Spaniards than like the other Arabic group. ${ }^{3}$

The spatial order measure followed closely the drawn model index. A one-way ANOVA on the proportion of valid L-R trials showed a significant effect of group, $F(2,58)=$ 5.36, $p=.007, \eta^{2}=.16$. Spanish participants tended to fill up the left space before the right space (a $72.6 \%$ preference for combinations LCR, CLR, LRC). In contrast, the Moroccan group showed the opposite preference (35.9\% of $\mathrm{L}-\mathrm{R}$ combinations - i.e., a preference for $\mathrm{R}-\mathrm{L}$ combinations RCL, CRL, RLC). The difference between the two groups was significant, $t(1,34)=3.33, p=.002$. Again, the comparison between the Spanish and the Arabs-in-Spain groups failed to be significant $(72.6 \%$ vs. $64.7 \%$, respectively), $t(1,42)=-0.69, p=.48$, whereas the Arabs-inSpain differed from the Moroccan group $(64.7 \%$ vs. $35.9 \%), t(1,40)=2.59, p=.01$.

We then proceeded to analyze the differences in preferred object orders. Here, we expected no cultural differences: In both cultures, participants should prefer the orders that impose a smaller working memory load. The results substantiated this prediction (see Fig. 2): All groups preferred

\footnotetext{
${ }^{3}$ Inspection of mean percentages for each sentence showed that the observed results were not due to atypical behavior in one or a few items. The range was 63.2-81.3 in the Spanish group, 25.0-56.3 in the Moroccan group, and 52.2-61.9 in the Arabs-in-Spain group. The highest and lowest items were not the same across groups.
} 
Table 2 Mean percentages of valid trials and standard errors (in brackets) in Experiment 1

\begin{tabular}{lrcc}
\hline & $\begin{array}{c}\text { Spaniards } \\
\text { (Spanish) }\end{array}$ & $\begin{array}{l}\text { Moroccans } \\
\text { (Darija) }\end{array}$ & $\begin{array}{l}\text { Arabs-in-Spain } \\
\text { (Spanish) }\end{array}$ \\
\hline L-R drawn model & $70.7(6.5)$ & $38.3(5.7)$ & $58.8(6.7)$ \\
L-R spatial order & $72.6(8.1)$ & $36(7.2)$ & $64.7(7.7)$ \\
LRC & $7.4(4.4)$ & $8.4(3.6)$ & $5.3(2.6)$ \\
LCR & $22.8(7.4)$ & $10.5(4.1)$ & $13.8(5.1)$ \\
CLR & $42.5(8.4)$ & $17.1(4.3)$ & $45.6(8.5)$ \\
CRL & $24.2(8.3)$ & $43.4(7.5)$ & $30.3(6.6)$ \\
RLC & $1.1(1.1)$ & $2.4(1.6)$ & $1.6(1.1)$ \\
RCL & $2.1(1.4)$ & $18.2(6.8)$ & $3.4(1.6)$ \\
Object order 123 & $58(7.6)$ & $45.2(6.7)$ & $56.8(6.9)$ \\
132 & $8.7(3.2)$ & $15.3(4)$ & $19.1(4)$ \\
213 & $18.6(6.3)$ & $19(6)$ & $12.2(4)$ \\
231 & $6.3(3.9)$ & $9.7(4.3)$ & $5(2.2)$ \\
312 & $4.2(1.9)$ & $7(2.8)$ & $6.1(2.6)$ \\
321 & $4.2(3.3)$ & $3.8(2.1)$ & $0.8(0.8)$ \\
\hline
\end{tabular}

Note. $\mathrm{L}=$ left; $\mathrm{R}=$ right; $\mathrm{C}=$ center

the 123 order, followed at a great distance by 132, after which the numeric value of proportions decreased progressively as memory load increased. Statistically, no significant differences were found between the three groups of participants on each of the possible orders (Kruskal-Wallis test with group as a factor, $N=65$; order $123, H=2.22, p=.33$; order $132, H=5.22, p=.07$; order $213, H=1.40, p=.49$; order $231, H=1.46, p=.48$; order $312, H=0.44, p=.80$; order $321, H=3.77, p=.15$ ). The analysis of the different orders shows that 123 was the preferred option. A contrast with the next most common (132) is highly significant, Mann-Whitney $U=1,640, p<.001$. The remaining orders are not significantly different from one another. Even comparing conditions 132 and 321, the difference is nonsignificant, $U=335.5, p=.062$. These results suggest that

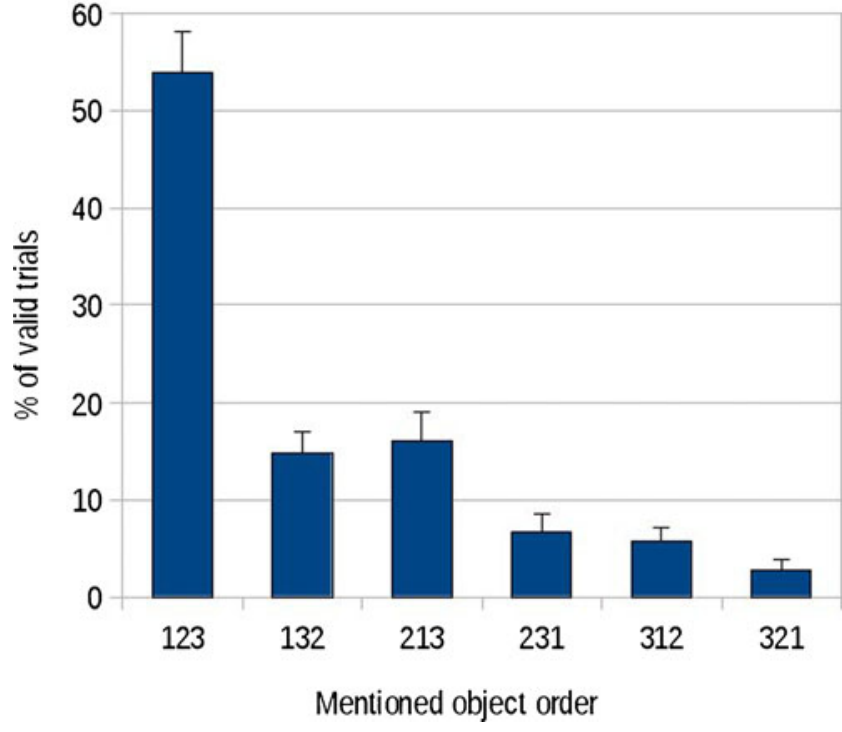

Fig. 2 Percentage of valid trials in each combination of object order. Error bars represent the standard error of the mean

participants are sensitive to the increase in memory load that occurs from order 123 to all other orders. The latter are all preferred to the same (low) extent. Present data and analyses do not support the existence of a gradation of preference, but this might be due to the very small proportions observed in those conditions.

\section{Discussion}

Readers of Spanish (an L-R language) and Standard Arabic $(\mathrm{R}-\mathrm{L})$, when tested in their native language and immersed in their own culture, differ in their preferred spatial choices when constructing mental models of static scenes from auditory linguistic input: Spanish readers prefer L-R models and Arabic readers prefer R-L models. Hence, effects of habitual RWD can be observed in static scenes, as well as in the intrinsically ordered events that have received attention
Fig. 1 Drawn model preference (percentages of valid trials) in each group. A value of $50 \%$ indicates no lateral bias.

Error bars represent the standard error of the mean

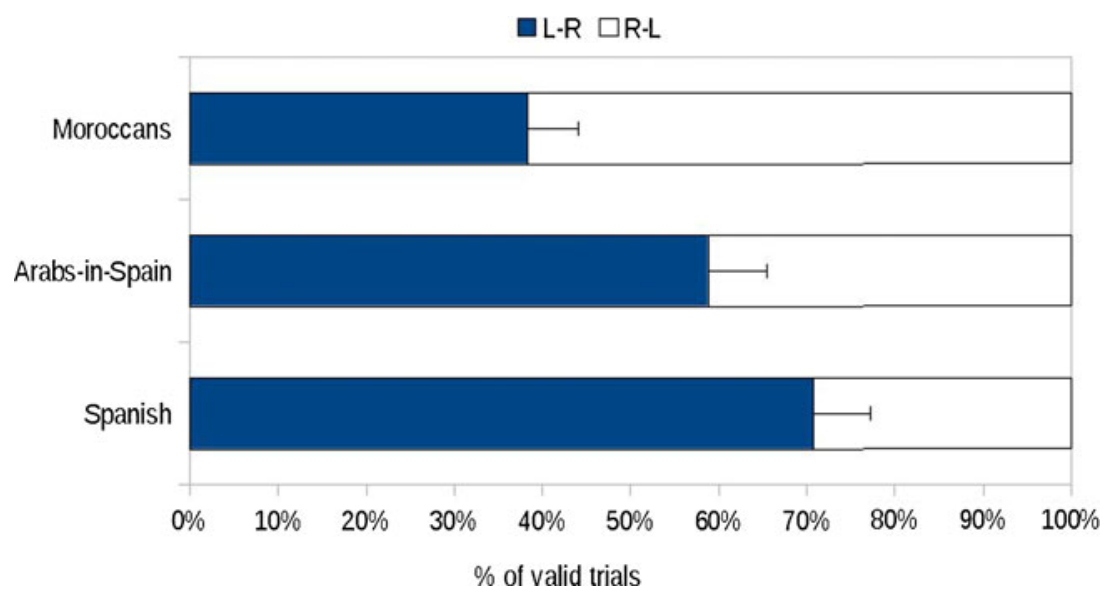


so far in the literature (see the introduction). Thus, it does not seem necessary that linguistic meaning have intrinsic order for it to be construed linearly in a spatial mental model in a systematic way. Present results also reveal that cultural differences in mental model construction due to RWD are confined to spatial preferences but that the management of working memory resources is similar in all groups. This check grants the use of the measure of directionality of the drawn model as an index of the directionality of the underlying mental model.

Present data are consistent with the proposal that lateral biases in mental model construction vary as a function of the degree of practice in a certain direction. The Moroccan participants, who were exposed to more inconsistent RWD habits than the Spanish participants, showed a weaker lateral bias (11.7 \% R-L vs. $20.7 \%$ L-R, respectively). Moreover, the Arabs-in-Spain group, who had been immersed in a $\mathrm{L}-\mathrm{R}$ culture and language for a long period of time, actually reversed their Arabic R-L bias and were statistically indistinguishable from the Spanish (although their L-R bias was also not as strong in magnitude: $8.8 \%$ vs. $20.7 \%$, both LR). However, since the two Arabic groups differed both in their degree of practice with $\mathrm{L}-\mathrm{R}$ scripts (greater in the Arabs-in-Spain group) and in the language used in the experiment (Spanish vs. Darija), there remains the possibility that any (or both) of these factors could be the main one responsible for the observed differences in lateral preferences. To disentangle these possibilities, we conducted a second experiment where a group of bilingual Moroccans were tested in their country using two languages of different script directionality. By keeping constant the participants and the cultural environment, we aimed to measure the influence of the input language. Since the task was repeated with a different language, we also aimed to see whether there were carryover influences from the first to the second language and whether they differed depending on whether the first language was the more or less practiced one.

\section{Experiment 2}

Method

\section{Participants}

A new group of 48 Moroccan students from the Abdelmalek Esaadi University, Tetouan, Morocco, were recruited (mean age, 23.5 years; 29 males; 1 left-handed and 1 ambidextrous). All the participants were born in Morocco and had never lived in a Western country. All of them were native speakers of Moroccan Darija (the local Arabic dialect), and 7 of them were also native speakers of Standard Arabic (starting before the age of 4 ). Nineteen of them were also highly fluent in French and 1 in Spanish, and 27 of them were quite fluent in both romance languages (started in high school or university). All but 3 were highly proficient and habitual readers of Standard Arabic. The remaining 3 also read Standard Arabic comfortably, but they did not do it on a daily basis.

\section{Procedure}

The procedure was essentially the same as that in Experiment 1 . The difference in this case was that each participant received two sets of five sentences. The first set was composed of the five sentences from Experiment 1, and the second set consisted of the following five new sentences:

6. The triangle is between the circle and the square.

7. The knife is between the fork and the spoon.

8. The phone is between the chair and the bed.

9. The wardrobe is between the door and the window.

10. The star is between the sun and the moon.

For each participant, one block was presented in Standard Arabic and the other block in a Romance language (either French or Spanish, both L-R languages) according to the preference of the participant. Half the participants started with a Romance language and moved to the Arabic language, and the others followed the opposite order. The mapping of sentence sets and languages to blocks was counterbalanced over participants, such that both sets and languages were presented an equal number of times in each block. Within each block, the sentences were presented in a fixed order.

\section{Results}

Criteria for exclusion of invalid trials were the same as those in the prior experiment. Three participants were discarded because all ten trials were invalid. There were no participants with all five trials invalid in one language but at least one valid trial in the other language. Out of the remaining 45 participants, the number of items rejected was $5.3 \%(6.2 \%$ in the $\mathrm{L}-\mathrm{R}$ language condition and $4.4 \%$ in the $\mathrm{R}-\mathrm{L}$ language condition).

Experiment 1 showed that there is a strong relation between spatial order and drawn model order and that object order (affected by working memory management processes) is independent of RWD. Hence, in this second experiment, we focused only on the directionality of the drawn models. Table 3 shows cell means and standard errors. The analysis proceeded in two stages. First, we focused only on the first block and compared the L-R Romance group with the $\mathrm{R}-\mathrm{L}$ Arabic group. The difference in means was in the right direction (58.6\% vs. $42.4 \%$; see Fig. 3), but the contrast 
Table 3 Mean percentages of valid trials and standard errors (in brackets) with an L-R drawn model in Experiment 2

\begin{tabular}{llc}
\hline & First block & Second block \\
\hline Romance (French or Spanish) & $58.6(6.6)$ & $37.9(7.2)$ \\
Standard Arabic & $42.4(7.7)$ & $50.6(6.5)$ \\
\hline
\end{tabular}

fell short of significance, $t(43)=-1.60, p=.11$. This might be due to lack of statistical power. One way to increase power for this particular contrast is to pool together the Arabic group in Experiment 2 with the Darija group in Experiment 1. These two groups are equivalent in all respects because they did the task in the same location and culture (Morocco) and their input language is written from right to left (actually, the written language is literally the same, Standard Arabic; see footnote 1). The increased number of observations in one of the samples yielded the contrast between L-R Romance and R-L Arabic significant, $t(60)=-2.19, p=.03$, suggesting that the directionality of the script of the input language is able to bias the directionality of mental model construction. Finally, it is also possible to pool together the Arab groups who did the task in a $\mathrm{L}-\mathrm{R}$ language in both experiments (the Arabs-in-Spain group in Experiment 1 and the Romance group in Experiment 2), although the groups were not so closely matched as the two Arabic groups who did the task in Arabic. When both pooled groups are compared, the effect of input language is highly significant, $t(85)=2.64, p=.009$.

Is there also an effect of the amount of practice with a script besides the immediate effect of the input language? One way to test this possibility is to compare the $\mathrm{L}-\mathrm{R}$ Romance group in Experiment 2 (Arabic readers who did the task in an L-R language) and the Arabs-in-Spain group in Experiment 1 (Arabic readers who also did the task in an L-R language, Spanish, after living immersed in Spanish culture for several years). These two groups yielded virtually identical L-R preferences ( $58.6 \%$ vs. $58.8 \%), t(46)=$ $0.01, p=.99$. Moreover, when these two groups were pooled together and compared with Spanish monolinguals, although their L-R bias was smaller (58.7\% vs. $70.7 \%)$, this difference still failed to be significant, $t(1,65)=-1.41, p$ $=.16$. This all runs against an influence of degree of practice with the script on lateral spatial biases (at least in participants who are already highly proficient in both scripts). Perhaps when two scripts of different directionality are practiced enough, such strategies become so fluid that they can be automatically activated by the input language and flipped over without any inertia.

However, Experiment 2 allowed a test of the potential carryover effects from one language to a subsequent language with a different written directionality, since the task was repeated in a second block with different materials in their other language. Thus, we could compare the proportion of $\mathrm{L}-\mathrm{R}$ models that were produced to a given input language when it was the first language in the experiment versus when it was preceded by a language of different directionality. Therefore, we could also assess whether the carryover effects differed in magnitude depending on whether they went from the most practiced to the less practiced language and script or the other way around.

The results showed clear carryover effects of different magnitude for each input language (see Fig. 4). When the task was done in a Romance language, the proportion of $\mathrm{L}-$ R models dropped from $58.6 \%$ to $37.9 \%$ when there was a preceding block with the same task in Arabic, $t(43)=2.13, p$ $=.03$. In other words, Arabic had a clear carryover effect on Romance. In contrast, when the task was done in Standard Arabic, the increase from $42.4 \% \mathrm{~L}-\mathrm{R}$ models (when done as a first block) to $50.6 \%$ (when preceded by Romance) was not significant, $t(43)=0.82, p=.41$. That is, Romance failed to have a carryover effect on Arabic. The most likely reason for this difference is that the Moroccan participants were more highly practiced reading Standard Arabic than their preferred Romance language (either French or Spanish). Therefore, carryover effects were stronger from the more practiced script to the less practiced script than in the
Fig. 3 The upper two bars (in dark gray) present the percentages of valid $\mathrm{L}-\mathrm{R}$ and $\mathrm{R}-\mathrm{L}$ models produced by Moroccan participants in the first experimental block, as a function of the input language: L-R Romance (either French or Spanish) or R-L Arabic. The lower three bars (in light gray) present the results of the single block in Experiment 1, reprinted here to ease crossexperiment comparisons. Error bars represent the standard error of the mean

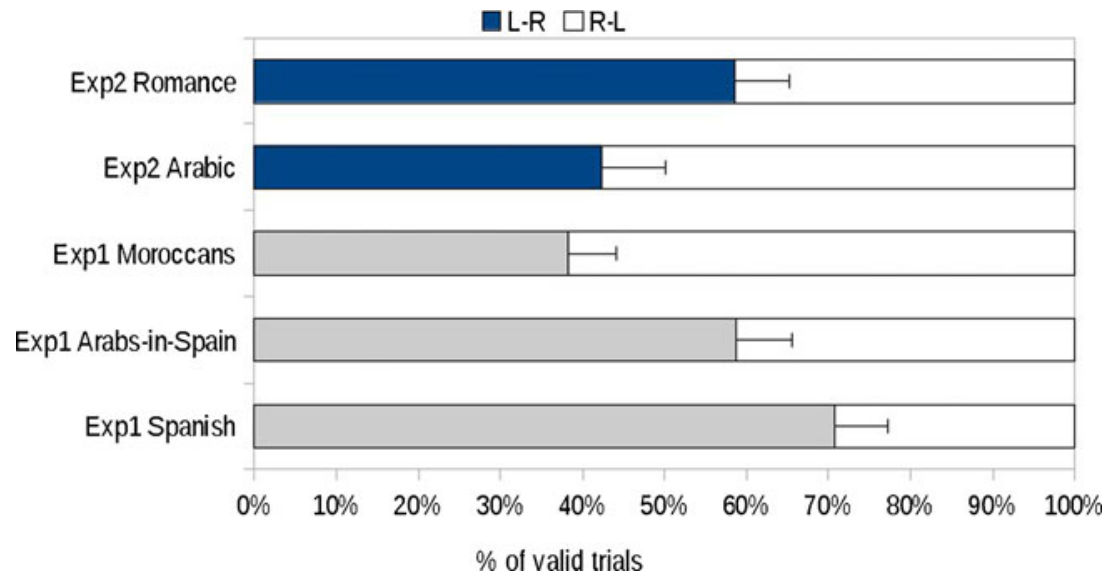


Fig. 4 Drawn model

preference (percentages of valid trials) as a function of the input language (L-R Romance or $\mathrm{R}-\mathrm{L}$ Arabic) when that language was presented in the first block versus when it was preceded by another block in a language of contrasting directionality. Error bars represent the standard error of the mean

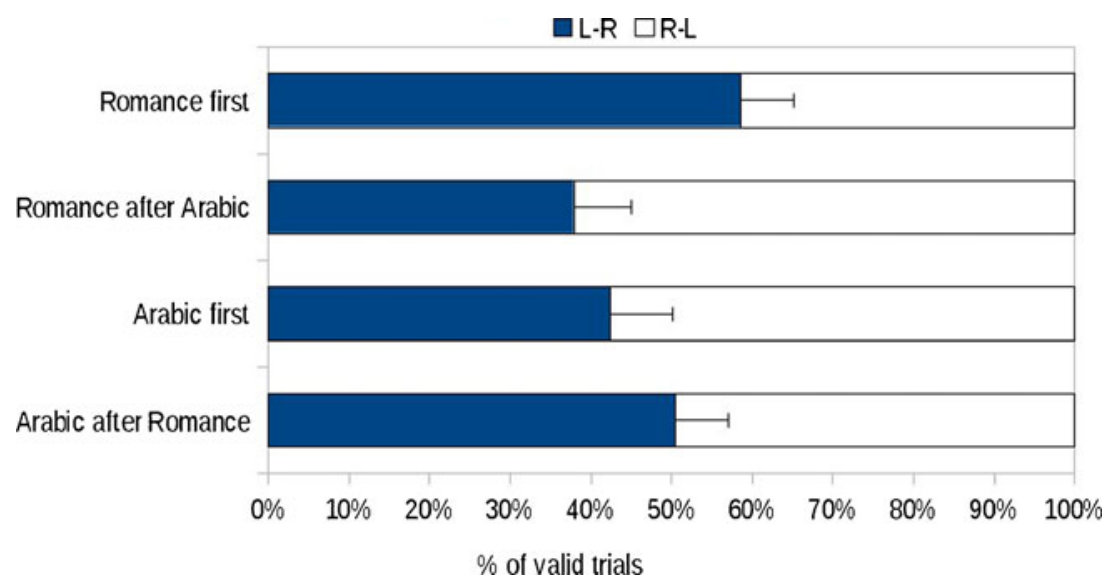

opposite direction. Extended practice in forming mental models with a given directionality does induce a long-term bias toward keeping that favorite directionality or going back to it more easily.

\section{General discussion}

The present study assessed the influence of RWD habits on mental model construction from auditory linguistic input. In order to tap more directly into the spatial components of those models that relate to RWD, we used descriptions of static scenes as input. The spatial configuration of mental models was inferred from how the scenes were drawn. Experiment 1 showed that Spanish monolingual participants, who read and write from left to right, tend to produce L-R models, whereas Moroccan participants, who mainly read and write in Standard Arabic from right to left (but also know at least one L-R language), show a (smaller) tendency to produce $\mathrm{R}-\mathrm{L}$ models. A group of Arabs who had been living in Spain for several years showed an L-R bias comparable to that of the Spanish group, although numerically smaller. Taken together, these results support an effect of RWD habits on mental model construction from language. This occurred in the context of identical strategies of working memory management in all three groups: a similar preference for the order of object production that minimized the number of items and time in memory.

However, because both the Spanish participants and the Arabs-in-Spain group heard the sentences in Spanish, whereas the Moroccan participants heard them in their local Arabic dialect, Experiment 1 was unable to disentangle two possible causes of the observed pattern of spatial biases: the long-term practice with a script versus the short-term influence of the language used as input in the task. In Experiment 2, Moroccan participants carried out the same task in either Standard Arabic or their preferred Romance language (either French or Spanish). When the input language was Standard Arabic, they behaved like the Moroccan group in the prior experiment. When the input language was French or Spanish, they behaved like the Arabs-in-Spain group in the prior experiment. Therefore, the results revealed a clear influence of the input language in spatial biases in mental model construction, suggesting a flexible deployment of spatial strategies depending on the language in use. The Arabic speakers' lateral bias when listening to an $\mathrm{L}-\mathrm{R}$ language was smaller in size than the bias shown by monolingual L-R Spanish readers, which suggests that there are no graded effects of conflicting bidirectional habits. However, the second block in Experiment 2 revealed carryover influences from the language used in the first block, and importantly, these influences varied depending on language. A previous block in Arabic had stronger carryover effects on a subsequent Romance block than vice versa. Although all participants were highly fluent bidirectional bilinguals, their degree of practice with the $\mathrm{R}-\mathrm{L}$ Arabic script was greater than with the L-R Roman script. Therefore, the pattern of carryover effects suggests an effect of degree of practice with the script in the face of highly flexible spatial strategies in language comprehension.

All in all, present results both support and qualify the standard view of the origin of RWD effects on high-level skills. In visual language comprehension, as word meanings are accessed, their referents are integrated into a developing mental model of the situation. Maass et al. (2009) and Chatterjee (2011) suggested that lateral biases at high levels of cognition arise because extended exposure to a given RWD leads to two effects, which may be causally linked between themselves or not. One is the development of lateral biases in perceptuo-motor habits such as scanning, exploration, and hand movements, which can also be applied to internal representations. Either as a consequence of these low-level biases or as an independent higher-level effect, there is also the establishment of a generalized schema for action that unfolds laterally in the same direction of the script. As a result, referents are placed in the mental model in a script-congruent way. This mental habit generalizes to situations where the linguistic input is not visual but 
auditory, as in the present study. It also generalizes more widely, reaching all kinds of concepts that are correlated with order of mention in language, such as causal relations in agent-patient interactions (Maass \& Russo, 2003) or temporal (Ouellet et al., 2010), numerical (Zebian, 2005) and letter (Shaki \& Gevers, 2011) sequences. If the pleasantness aroused by visual artistic depictions depends at least partially on their perceived naturalness, this account should also be able to explain the observed effects of script direction on aesthetic preferences (Pérez González, 2011).

Under this view, practice with directionally inconsistent scripts leads to the development of inconsistent habits and, thus, to competing tendencies of lateral mental model construction. The lateralized schema for action should grow stronger as congruent experiences accumulate, but weaker as conflicting experiences accumulate. Therefore, bidirectional bilinguals should show smaller lateral biases than should monolinguals. Many studies support this view (see Santiago \& Román, 2012). Present results add to this line of evidence with the finding of a small effect of amount of practice with the script.

However, contrary to the standard view, the clearest finding in the present study supports a very flexible deployment of mental lateral habits in mental model construction during language comprehension as a function of the input language in the experimental task. This evidence comes to join a recent strand in the literature that suggests that bidirectional bilinguals can acquire two contrasting lateral biases and flexibly show either one depending on the characteristics of the situation (e.g., the input language). Such flexible switching has been shown both on low-level skills, such as saccadic eye movements or foveal letter perception (Nazir, Ben-boutayab, Decoppet, Deutsch, \& Frost, 2004), and high-level representations, such as number and letter sequences (Fischer, Shaki, \& Cruise, 2009; Shaki \& Gevers, 2011), but not always (see Dehaene et al., 1993; see Santiago \& Román, 2012, for a review). Even more problematic for the standard view are studies that show that just a very small amount of practice with text that provides the opposite directional experiences can lead to complete reversals in lateral biases (see Fischer, Mills, \& Shaki, 2010, for numbers; see Shaki \& Gevers, 2011, for letter sequences; and see Casasanto \& Bottini, 2010, for time; for an extended discussion, see Santiago \& Román, 2012). Thus, people can sometimes switch flexibly and automatically from one spatial strategy to another, and an extended degree of practice with both strategies may not always be a requisite.

We suggest that, in order to account for this conflicting pattern of results, an alternative view may be helpful. The view we favor is an extension of the coherent working models theory put forward by Santiago et al. (2011). It rests on two assumptions. First, as people become more practiced readers, they develop a strong habit of representing language visually, independently of whether it has been presented in the visual or the auditory modality. Second, following universal working memory management strategies, the referred contents are accessed and introduced in the model in the order in which they are mentioned. Third, the contents of the model are laid out in the same direction as the representation of the input words due to a characteristic of mental models that has often not been emphasized enough: their push for internal consistency. Perhaps because creating mental models is intrinsically effortful, mental models are constrained to be as internally coherent and simple as possible, given the requirements of the task at hand. We have proposed elsewhere that this characteristic of mental models is central to accounting for the flexibility of conceptual congruency effects in a wide variety of tasks (Santiago, Ouellet, Román, \& Valenzuela, 2012; Santiago et al., 2011). If we assume that both the spatial representation of the input sentence and the mental model of its contents are parts of a superordinate mental model of the whole situation, both entities will tend to unfold in the same direction, because this will increase the internal coherence of the superordinate model. Because the directionality of the linguistic input is constrained by its entrenched association with written language (whose direction is fixed by the script), it is the directionality of the mental model that will adapt.

If this is so, the directionality of the mental model should depend strongly on the directionality of the language from which the model is being constructed. Changes in the spatial characteristics of the input language may induce very fast remappings of the mental model. Contextual priming, such as the language used for instructions, stimuli, or interaction with the experimenter, should also be able to affect the choice of spatial strategy in conceptual tasks with very little linguistic component, such as those that assess causal, number, and time representations.

This account is not incompatible with the development of long-term preferencies for one or another lateral strategy. Bidirectional bilinguals will gain extended practice in constructing models in both directions, developing two contextually dependent lateral schemas. Characteristics of the situation will control which of the two schemas will be activated and applied on a given occasion. Unbalanced bidirectional bilinguals may generate differential preferences for the use of one or another lateral strategy, and monolinguals are likely to generate a marked preference for the lateral strategy that matches their familiar script.

However, this view is in need of further development in order to provide an integrated account of available data. It will be necesary to specify in detail what the factors are that determine the predominance of short-term factors, such as input language, or long-term factors, such as degree of experience with a script, in a given task and context. So 
far, it is unclear why sometimes lateral habits are so flexible and require so little practice, whereas other times they develop slowly and are sensitive to the total amount of practice received in each direction throughout years of reading and writing experience. Given the number of different skills and representations that are affected by RWD, the complexity of the results reported so far, and the amount of lacunae that still remain to be filled (Santiago \& Román, 2012), this goal still seems far ahead. Our main tenet here is that a more flexible view of the effects of RWD, such as the one just sketched, will probably prove to be a more adequate theoretical tool than prior views that emphasize integration of reading experiences in a single lateralized schema for action.

To sum up, RWD is able to induce directional lateral biases in mental model construction during language comprehension. These spatial biases can be automatically and quite flexibly activated by the current linguistic input, but they also show a small degree of inertia that suggests that they can slowly build up with practice. The challenge for the future is to integrate these two apparently conflicting characteristics into an integrated theoretical account.

Author Note This research was supported by Grants P09-SEJ-4772, Junta de Andalucía and European Regional Development Fund (ERDF), and PSI2012-32464, Spanish Ministery of Economy and Competitivity, both to Julio Santiago (PI). We gratefully acknowledge the assistance in Morocco by Omar Badessi, Tarik Boubker, Bouthaina Ben El Amin, and Mouad El Mejdki. We are also grateful to the members of the Psycholinguistics group at the University of Granada and to Gabriella Vigliocco and the Language Processing Lab at University College, London, where the senior author spent a sabbatical year while this article was written, funded by the Spanish Ministery of Education (ref. PR2010-0283).

\section{References}

Altmann, L. J. P., Saleem, A., Kendall, D., Heilman, K. M., \& Rothi, L. J. G. (2006). Orthographic directionality and thematic role illustration in English and Arabic. Brain and Language, 97(3), 30616.

Ariel, R., Al-Harthy, I. S., Was, C. A., \& Dunlosky, J. (2011). Habitual reading biases in the allocation of study time. Psychonomic Bulletin \& Review, 18(5), 1015-21.

Barrett, A. M., Kim, M., Crucian, G. P., \& Heilman, K. M. (2002). Spatial bias: Effects of early reading direction on Korean subjects. Neuropsychologia, 40(7), 1003-12.

Berch, D. B., Foley, E. J., Hill, R. J., \& Ryan, P. M. (1999). Extracting parity and magnitude from arabic numerals: Developmental changes in number processing and mental representation. Journal of Experimental Child Psychology, 74, 286-308.

Casasanto, D. J., \& Bottini, R. (2010). Can mirror-reading reverse the flow of time? In C. Hölscher, T. F. Shipley, M. Olivetti Belardinelli, J. A. Bateman, \& N. S. Newcombe (Eds.), Spatial Cognition VII (pp. 335-345). Springer: Berlin Heidelberg

Chatterjee, A. (2011). Directional asymmetries in cognition: What is left to write about? In A. Maass \& T. W. Schubert (Eds.), Spatial dimensions of social thought (pp. 189-210). Berlin: Mouton de Gruyter.

Chatterjee, A., Southwood, M. H., \& Basilico, D. (1999). Verbs, events and spatial representations. Neuropsychologia, 37(4), 395-402.

Chokron, S., \& De Agostini, M. (2000). Reading habits influence aesthetic preference. Cognitive Brain Research, 10(1-2), 4549.

Chokron, S., \& Imbert, M. (1993). Influence of reading habits on line bisection. Cognitive Brain Research, 1(4), 219-22.

Dehaene, S., Bossini, S., \& Giraux, P. (1993). The mental representation of parity and number magnitude. Journal of Experimental Psychology: General, 122(3), 371-396.

Dobel, C., Diesendruck, G., \& Bölte, J. (2007). How writing system and age influence spatial representations of actions: A developmental, cross-linguistic study. Psychological Science, 18(6), 487491.

Evans, J. S. B. T., Newstead, S. E., \& Byrne, R. M. J. (1993). Human reasoning: The psychology of deduction. Hove, UK: Lawrence Erlbaum.

Fischer, M. H., Mills, R., \& Shaki, S. (2010). How to cook a SNARC: Number placement in text rapidly changes spatial-numerical associations. Brain and Cognition, 72(3), 333-336.

Fischer, M. H., Shaki, S., \& Cruise, A. (2009). It takes just one word to quash a SNARC. Experimental psychology, 56(5), 361-6.

Fuhrman, O., \& Boroditsky, L. (2010). Cross-cultural differences in mental representations of time: Evidence from an implicit nonlinguistic task. Cognitive Science, 34(8), 1430-1451.

Geminiani, G., Bisiach, E., Berti, A., \& Rusconi, M. L. (1995). Analogical representation and language structure. Neuropsychologia, 33(11), $1565-1574$.

Goodwin, G. P., \& Johnson-Laird, P. N. (2005). Reasoning about relations. Psychological Review, 112(2), 468-493.

Grice, H. P. (1975). Logic and conversation. In P. Cole, \& J. L. Morgan (Eds.), Syntax and semantics, vol.3, speech acts (vol. 3, pp. 4158). Academic Press.

Jahn, G., Knauff, M., \& Johnson-Laird, P. N. (2007). Preferred mental models in reasoning about spatial relations. Memory \& cognition, 35(8), 2075-87.

Johnson-Laird, P. N. (1983). Mental models. Cambridge, MA: Cambridge University Press.

Kugelmass, S., \& Lieblich, A. (1970). Perceptual exploration in Israeli children. Child Development, 41(4), 1125-1131.

Lewis, P. (Ed.). (2009). Ethnologue: Languages of the world. SIL International. Retrieved from http://www.ethnologue.com/

Maass, A., Pagani, D., \& Berta, E. (2007). How beautiful is the goal and how violent is the fistfight? Spatial bias in the interpretation of human behavior. Social Cognition, 25(6), 833-852.

Maass, A., \& Russo, A. (2003). Directional bias in the mental representation of spatial events: Nature or culture? Psychological Science, 14(4), 296-301.

Maass, A., Suitner, C., Favaretto, X., \& Cignacchi, M. (2009). Groups in space: Stereotypes and the spatial agency bias. Journal of Experimental Social Psychology, 45(3), 496-504.

Mishkin, M., \& Forgays, D. G. (1952). Word recognition as a function of retinal locus. Journal of Experimental Psychology, 43(1), 4348.

Nachshon, I. (1985). Directional preferences in perception of visual stimuli. The International Journal of Neuroscience, 25(3-4), $161-174$.

Nachshon, I., Argaman, E., \& Luria, A. (1999). Effects of directional habits and handedness on aesthetic preference for left and right profiles. Journal of Cross-Cultural Psychology, 30 (1), 106-114.

Nazir, T. A., Ben-boutayab, N., Decoppet, N., Deutsch, A., \& Frost, R. (2004). Reading habits, perceptual learning, and recognition of printed words. Brain and Language, 88, 294-311. 
Ouellet, M., Santiago, J., Israeli, Z., \& Gabay, S. (2010). Is the future the right time? Experimental psychology, 57(4), 30814.

Pollatsek, A., Bolozky, S., Well, A. D., \& Rayner, K. (1981). Asymmetries in the perceptual span for Israeli readers. Brain and Language, 14(1), 174-180.

Pérez González, C. (2011). Lateral organisation in nineteenth-century studio photographs is influenced by the direction of writing: A comparison of Iranian and Spanish photographs. Laterality. doi:10.1080/1357650X.2011.586701

Santiago, J., Ouellet, M., Román, A., \& Valenzuela, J. (2012). Attentional factors in conceptual congruency. Cognitive Science, in press. doi:10.1111/j.1551-6709.2012.01240.x

Santiago, J., Román, A., \& Ouellet, M. (2011). Flexible foundations of abstract thought: A review and a theory. In A. Maass \& T. W. Schubert (Eds.), Spatial dimensions of social thought (pp. 41110). Berlin: Mouton de Gruyter.

Santiago, J., \& Román, A. (2012). Reading and writing direction effects on cognition and aesthetics: Where do we stand? Manuscript submitted for publication.
Shaki, S., \& Gevers, W. (2011). Cultural characteristics dissociate magnitude and ordinal information processing. Journal of Cross-Cultural Psychology, 42(4), 639-650.

Shanon, B. (1979). Graphological patterns as a function of handedness and culture. Neuropsychologia, 17(5), 457-465.

Spalek, T. M., \& Hammad, S. (2005). The left-to-right bias in inhibition of return is due to the direction of reading. Psychological Science, 16(1), 15-18.

Tversky, B., Kugelmass, S., \& Winter, A. (1991). Cross-cultural and developmental trends in graphic productions. Cognitive Psychology, $23,515-557$

Van Dijk, T. A., \& Kintsch, W. (1983). Strategies in discourse comprehension. New York: Academic Press.

Zebian, S. (2005). Linkages between number concepts, spatial thinking, and directionality of writing: The SNARC effect and the reverse SNARC effect in English and Arabic monoliterates, biliterates, and illiterate Arabic speakers. Journal of Cognition and Culture, 1(2), 165-190.

Zwaan, R. A., \& Radvansky, G. A. (1998). Situation models in language comprehension and memory. Psychological Bulletin, 123 (2), 162-185. 